\title{
Variable star monitoring in local group dwarf irregular galaxies
}

\author{
Jan Snigula, Claus Gössl, Ulrich Hopp, Heinz Barwig \\ Universitäts-Sternwarte München, Scheiner Straße 1, D 81679 \\ München, Germany
}

\begin{abstract}
Dwarf galaxies in the local group provide a unique astrophysical laboratory. Despite their proximity some of these systems still lack reliable distance determinations as well as studies of their stellar content and star formation history. We present first results of our survey of variable stars in a sample of six local group dwarf irregular galaxies. Taking the Leo A dwarf galaxy as an example we describe observational strategies and data reduction. We discuss the light curves of two newly found Cepheids and place them into the context of a previously derived P-L relation. Finally we discuss the LPV content of Leo A.
\end{abstract}

\section{Introduction}

A magnitude-limited complete census of variable stars in nearby dwarf galaxies allows important contributions to the star formation history of these systems. Measurements of some variable stars can supply improved distance determinations for the host galaxies, others will provide important constraints for the population analysis. Different classes of variables can further improve the understanding of the star formation history of these system, functioning as tracers of star formation during different epochs. We expect the data set of our longterm monitoring program to be especially well suited to study the contents of red long-period variables and to re-investigate the paucity of Cepheids with $P>10 \mathrm{~d}$ as reported by Sandage \& Carlson (1985).

\section{Observations and data reduction}

We selected a sample of six local group dwarf irregular galaxies which are visible with the 0.8-m telescope of our institute at Mt Wendelstein. The names and additional data from the literature compilation by Mateo (1998) are shown in Table 1.

The observations so far were carried out in $R$ - and $B$-band, sparsely sampling a 3-yr period starting with test observations in 1999. This part of the data set should be sensitive to long-period variable stars with periods up to $\sim 500 \mathrm{~d}$. Additional observations in $R-, B$ - and $I$-band were obtained during 3 observing campaigns at the $1.23-\mathrm{m}$ telescope on Calar Alto densely sampling three two-week-long periods. These observations should provide grounds for a search for variable stars with shorter periods ranging from $\sim 1.5 \mathrm{~d}$ up to $\sim 10 \mathrm{~d}$. 
Table 1. Names, variable star counts, absolute $B$-band brightness in mag, and current distance estimation in $\mathrm{kpc}$ for the dwarf galaxies observed in our project. The data are taken from the literature compilation by Mateo (1998). For Leo A the data are from the work of Dolphin et. al (2002) and from this work.

\begin{tabular}{ll|ccccc}
\hline & & RR Lyr & $\delta$ Cep & LPV & $M_{B}$ & distance \\
\hline LGS 3 & (Pisces) & - & - & - & -9.9 & $810 \pm 60$ \\
UGCA 92 & (EGB 0427+63) & - & - & - & -11.6 & $1300 \pm 700$ \\
DDO 69 & (Leo A) & 8 & $66+1^{1}$ & $16^{1}$ & -11.3 & $690 \pm 100$ \\
DDO 155 & (GR 8) & - & $1 ?$ & $5 ?$ & -11.2 & $1590 \pm 600$ \\
DDO 210 & (Aquarius) & - & 0 & - & -9.9 & $800 \pm 250$ \\
DDO 216 & (Pegasus) & - & $7-10$ & - & -12.3 & $955 \pm 50$ \\
\hline
\end{tabular}

This work

The acquired data were bias subtracted, flat-fielded and cosmic ray rejected. Then, the images from one night were astrometrically aligned to a common reference frame and combined with individual weights proportional to their $S / N$. For each epoch, consisting of all the stacked images of a single night, a difference image against a common deep reference frame was created using an implementation (Gössl \& Riffeser 2002, 2003) of the Alard algorithm (Alard \& Lupton 1998). Finally, these difference images were convolved with a stellar PSF.

To extract light curves from the reduced data, first all pixels deviating significantly $(3 \sigma)$ from the reference image in a minimum number of epochs $n$ were flagged, utilizing the complete per-pixel error propagation of our data reduction pipeline. Then, using these coordinates as input, values and associated errors were read from the difference images and the light curve data were assembled. To search for periodic signals in the extracted difference fluxes, a Lomb (1976) algorithm using the interpretation from Scargle (1982) was applied.

The photometric calibration was conducted using the HST data published by Schulte-Ladbeck et al. (2003).

\section{Preliminary results}

For the galaxies Leo A and UGCA 92 we have very good monitoring and a large fraction of the data already has passed the pipeline. The Leo A data set serves as a test case: A total of 26 variable star candidates were detected. Among them, we identified 16 secure long period variables (LPV) (typical average values $19.4<$ $R<22.1$, and $74 \mathrm{~d}<\mathrm{P}<590 \mathrm{~d}$ ), and we have 8 further candidates for LPVs. In addition, we were able to identify two good candidate Cepheids with best-fitting periods of 6.4 and $1.69 \mathrm{~d}$. The later candidate was previously described by Dolphin et al. (2002) as C2-V58 with a period of $1.4 \mathrm{~d}$. The Dolphin et al. period solution fails in deriving a reliable light curve with our data, yet applying our period value to their data set yields reasonable results. The phase convolved light curves for the two Cepheids are shown in Fig. 1. 

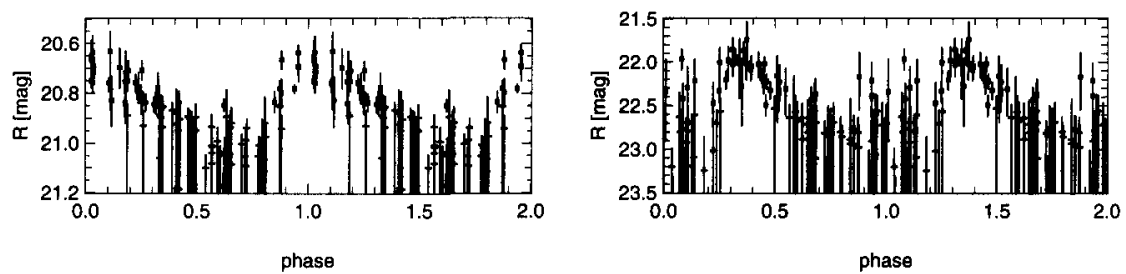

Figure 1. Phase convolved light curves for two Cepheids found in the Leo A dwarf galaxy. Plotted are the apparent $R$-band magnitudes against the phase. The left panel shows a Cepheid with a 6.4-d period. In the right panel a Cepheid with a period of $1.69 \mathrm{~d}$ is shown that was previously published by Dolphin et al. (2002) with a period of $1.4 \mathrm{~d}$.

\section{Comparison with published work}

The color-magnitude diagram shown in the left panel of Fig. 2 is based upon the HST data published by Tolstoy et al. (1996) and Schulte-Ladbeck et al. (2002). Flagged by bigger symbols are those variables from our sample that lie inside the HST field of view, two Cepheids in the instability strip (crosses) and the candidates for long term variability (triangles) in the regime of the red giants.

Tolstoy et al. (1996) found from ground-based data a distance modulus for Leo A of 24.2 and a resulting distance of $690 \mathrm{kpc}$ (see also Schulte-Ladbeck et al. 2002). This result got further support from the search for short period variables with the WIYN telescope over 3 consecutive days in 2000 December (Dolphin et al. 2002). Our data complement this dataset for longer periods.

The right hand panel of Fig. 2 shows the period-luminosity (PL) relation of the SMC shifted to the distance determined by Tolstoy et al. The short period variables measured by Dolphin coincide with the shown PL relation. The overplotted values for the two Cepheids from our survey (crosses) support this relation also in the regime of longer periods.

\section{Summary}

We presented preliminary results for our survey for variable stars in a sample of irregular local group dwarf galaxies. For the Leo A dwarf galaxy, the best analysed case so far, we already identified a total of 26 candidates for variability, 16 of these as long period variables and two Cepheids. We compared the latter with the period-luminosity relation and the short period variables discussed by Dolphin et al. (2002). We found that our Cepheids fully support their findings and the resulting distance estimate for Leo A. This result is further in good agreement with the TRGB distance (Tolstoy et al. 1996, Schulte-Ladbeck et al. 2002). The location of the LPVs in the color-magnitude diagram indicate that most of them are early asymptotic giant branch stars. While a complete census of these intermediate age stars is missing for most of the Local Group members, a proper statistic of their appearance can guide the reconstruction of the star formation history at the age of several Gyr, by-passing the agemetallicity degeneracy inherent in color-magnitude diagram studies. 

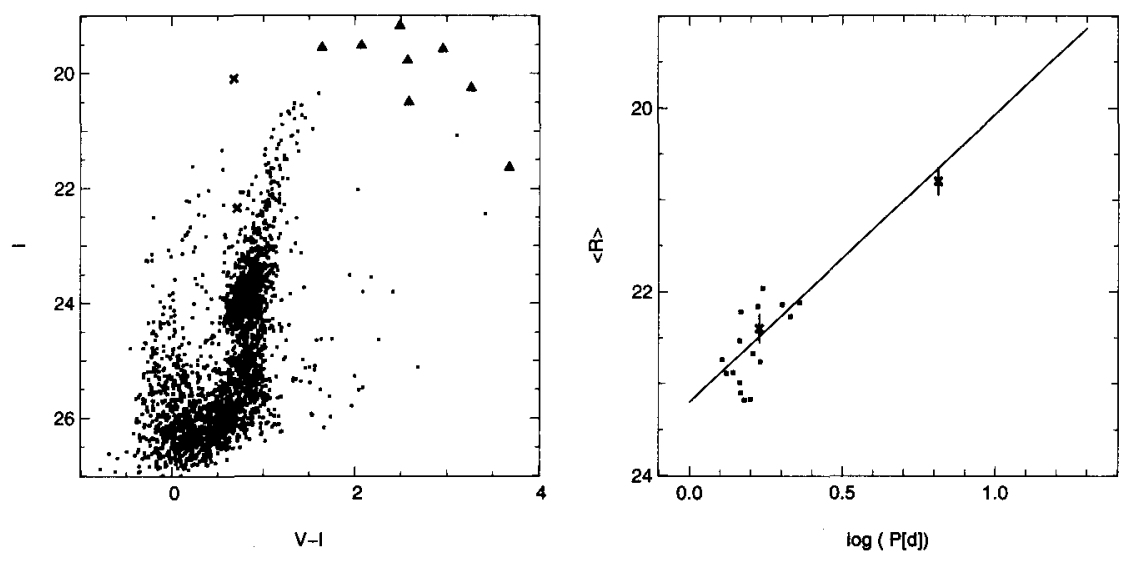

Figure 2. The left hand panel shows a color-magnitude diagram based on HST observations by Schulte-Ladbeck et al. (2003). The discovered variables in Leo A with positions falling into the field of view of the HST observations are plotted as triangles. The right hand panel shows the period-luminosity relation of the SMC shifted to the distance determined by Tolstoy et al. (1996). The small dots mark the Cepheids found by Dolphin et al. (2002), the large crosses the two Cepheids presented here.

Acknowledgments. We wish to thank Drs I. Drozdovsky, C. Maraston, R.E. Schulte-Ladbeck, and E. Tolstoy for helpful discussions. We acknowledge the support of the Calar Alto and Wendelstein staff. J. Fliri and A. Riffeser carried out some of our observations. The project is supported by the Deutsche Forschungsgemeinschaft grant Ho 1812/3-1 and Ho 1812/3-2.

\section{References}

Alard, C., Lupton, R.H. 1998, ApJ, 503, 325

Dolphin, A.E., et al. 2002, AJ, 123, 3154

Gössl C.A., Riffeser, A. 2002, A\&A, 381, 1095

Gössl, C.A., Riffeser, A. 2003, ASP Conf. Ser. 295, 229

Lomb, N.R. 1976, Ap\&SS, 39, 447

Mateo, M.L. 1998, ARA\&A, 36, 435

Sandage, A., Carlson, G. 1985, AJ, 90, 1464

Scargle, J.D. 1982, ApJ, 263, 835

Schulte-Ladbeck, R., et al. 2002, AJ, 124, 896

Tolstoy, E., et al. 1996, AJ, 116, 1244 


\section{Discussion}

Dambis: Are there any red supergiants among the LPVs discovered? Have you tried to cross-identify your variables with 2MASS (red supergiants are above the detection limit of 2MASS)?

Snigula: We have not yet tried to cross-identify our sources with objects in the 2MASS catalog, but that is something we definitely plan to do.

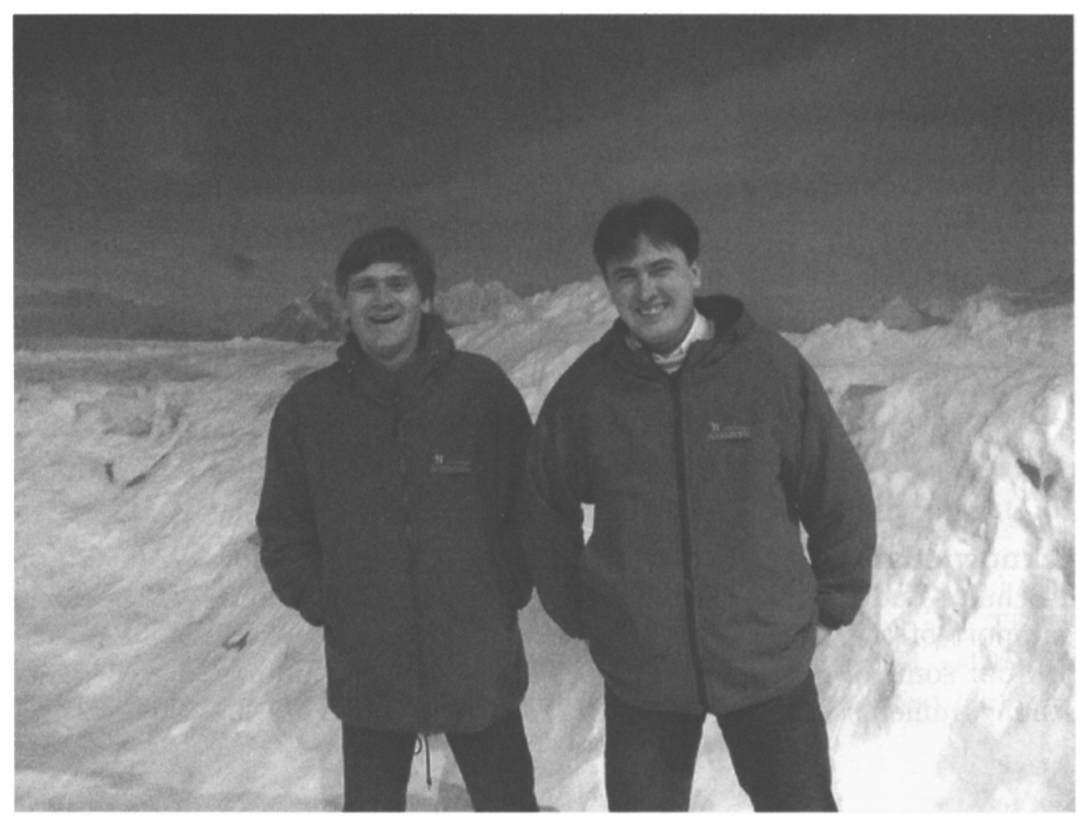

Andrei Dambis and Mikhail Sachkov 